\title{
Interstellar polarization at high galactic latitudes from distant stars $^{\star}$
}

\section{A catalog of polarization data for the North Galactic Pole Area}

\author{
A. Berdyugin ${ }^{1}$, P. Teerikorpi ${ }^{1}$, L. Haikala ${ }^{2}, 3$, M. Hanski ${ }^{1}$, J. Knude $^{4}$, and T. Markkanen ${ }^{3}$ \\ 1 Tuorla Observatory, 21500 Piikkiö, Finland \\ e-mail: pekkatee@astro.utu.fi; mhanski@astro.utu.fi \\ 2 European Southern Observatory, Casilla 19001, Santiago, Chile \\ e-mail: Ihaikala@eso.org \\ 3 Observatory and Astrophysics Laboratory, PO Box 14 00014, Helsinki University, Finland \\ e-mail: tapio.markkanen@helsinki.fi \\ 4 Niels Bohr Institute for Astronomy, Geophysics and Physics, Juliane Maries Vej 32, 2100 Kobenhavn O, \\ Denmark \\ e-mail: indus@astro.ku.dk
}

Received 11 December 2000 / Accepted 5 March 2001

\begin{abstract}
We present a catalog of interstellar polarization measurements for the North Galactic Pole area $(b>$ $70^{\circ}$ ). Our list contains 336 stars of spectral classes from A to $\mathrm{K}$ for which the linear polarization has been measured by us and other researchers. The data are useful for investigation of the distribution of dust, to estimate the amount of interstellar extinction and to map Galactic magnetic field at high galactic latitudes. We discuss also the data selection, sampling and completeness of the catalog.
\end{abstract}

Key words. polarization - dust, extinction - solar neighbourhood

\section{Introduction}

The present paper presents and discusses data which have been used in our series on interstellar polarization at high northern galactic latitudes (by Berdyugin et al. 1995, Paper I, Berdyugin \& Teerikorpi 1997, Paper II; Berdyugin et al. 2000, Paper III). We also briefly discuss such questions as the completeness of the catalog and make a comparison of the photometric distances with those from HIPPARCOS, when available.

The aim of our research programme is to investigate the interstellar polarization in the North Galactic Pole (NGP) region $\left(b>70^{\circ}\right)$ from observations of stars at intermediate and large distances $(d>300 \mathrm{pc})$ and compare these measurements with those obtained for more nearby stars. These results are applied for the determination of the amount of interstellar extinction and the structure of the Galactic magnetic field in this area; Papers I

\footnotetext{
Send offprint requests to: A. Berdyugin, e-mail: andrei@astro.utu.fi

* Table 1 is only available in electronic form at the CDS via anonymous ftp to

cdsarc.u-strasbg.fr (130.79.128.5) and

http://cdsweb.u-strasbg.fr/cgi-bin/qcat?/A+A/372/276

or via http://www. edpsciences.org
}

and II were mainly concerned with the distance dependence of polarization, while in Paper III we studied in detail the distribution of the polarization directions over the NGP, complementing the data in Papers I and II with our more recent unpublished measurements and older measurements of closer stars made by other researchers. In the present paper we give a table of polarization data upon which Papers I-III were based. This catalog also shows the present level of polarization measurements over the NGP and forms a useful basis for futher observations.

\section{The sample of stars and data selection}

The first star sample in the Crimea-Tuorla-NOT programme was based on the photometric catalog by Hill et al. (1982), listing A-F stars in the region of the NGP $\left(b>75^{\circ}\right)$. The polarization data for the brightest stars were obtained with the $60 \mathrm{~cm}$ telescope of Tuorla Observatory and with the $1.25 \mathrm{~m}$ telescope of the Crimean Astrophysical Observatory (52 stars, Paper I).

The next sample of more distant and fainter stars was observed with the $2.6 \mathrm{~m}$ Nordic Optical Telescope (NOT), La Palma (20 stars, Paper II). Starting from 1997 we have complemented our sample with stars from the unpublished 
list by Knude (see e.g. Knude 1996). With the NOT we have obtained polarization data for 94 more stars. The details of our observation techniques and data reduction can be found in Papers I and II.

In addition, we give the older polarization measurements of the stars in the NGP area made by Markkanen (1979) and Haikala (1979). We also include in our list polarization data for high latitude stars $\left(b>70^{\circ}\right)$ selected from the polarization catalogs published by Appenzeller (1968), Mathewson \& Ford (1970) and Korhonen \& Reiz (1986). These measurements concern primarily bright and nearby stars.

Selecting the stars from such different sources we have used the same accuracy criterium: only the data with $P>2 \sigma_{\mathrm{P}}$ are taken. Under this restriction the error in the derived direction angle of polarization is $\leq 14^{\circ}$. To deal with very little or apparently non-polarized stars we have assumed that if $P$ and $\sigma_{\mathrm{P}}<0.05$ then $P=0.0$. As in Appenzeller's catalog (1968) polarization and its error are given in stellar magnitudes, we have used the formula $P(\%)=P(\mathrm{mag}) \times 46.05$ to make a convertion. Mathewson $\&$ Ford (1970) did not give the error of measurement in their data table, but they gave an analytical expression which we have used to calculate it.

There are about 20 stars in our list for which the polarization has been measured by more than one astronomer. In general, the agreement in polarization magnitude and position angle of polarization vector between different papers and different authors is good, with a few exeptions, for which we make special notes. Whenever this happens, the main entry in our table gives those polarization data for which the reported measuring accuracy is the highest. As polarization variables might be among those stars, we have excluded them from the polarization maps.

\subsection{Distance estimates}

At the time when the older measurements were done and our own programme was started, only photometric distances were available for most of the stars in the studied area. Presently most of the stars closer than 400 pc in the NGP region have accurate parallaxes measured by the HIPPARCOS astrometrical satellite (Perryman et al. 1997). Whenever possible, we give the distances derived from parallaxes (when $\pi>2 \sigma_{\pi}$ ). Thus for about $90 \%$ of the stars closer than $300 \mathrm{pc}$ the distance estimates come from the parallax, while for more distant stars $(d>$ $400 \mathrm{pc}$ ) the estimates are mostly from photometry made either by Hill et al. (1982) or Knude (1996). There are several stars in the list for which the distances are not available.

Figure 1 shows three-dimensional plots of the stars in the NGP area with measured polarization. We should point out that only by the means of a large telescope, i.e. with the NOT, it became possible to go beyond $Z \approx$ 300 pc up to $Z=1500$ pc. a)

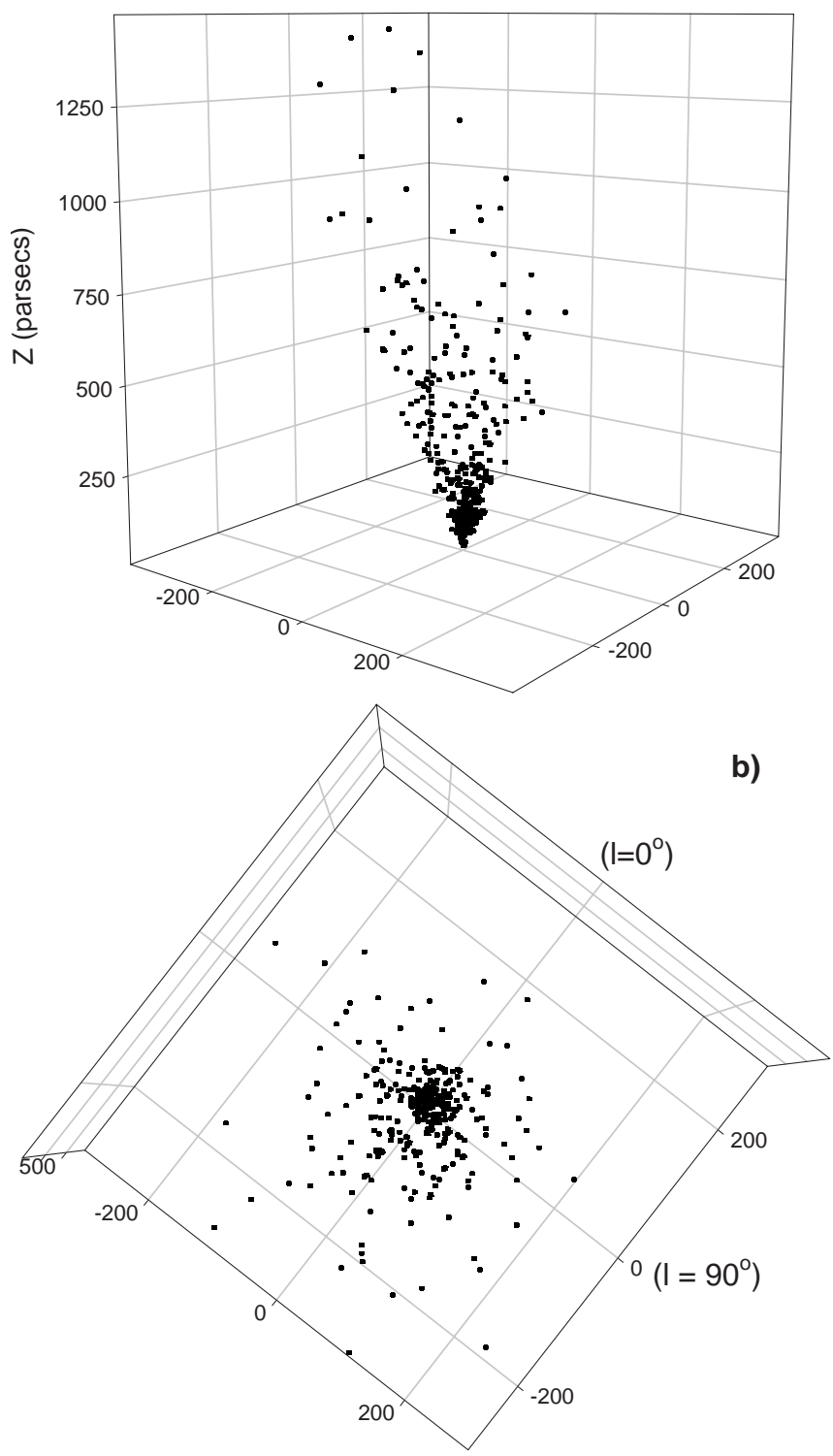

Fig. 1. Three-dimensional plots of the stars in the NGP area with measured polarization. The Sun is in the origin of coordinate axes, $Z$-distance increases upward. a) $Z$-axis wiev, b) $X Y$-plane wiev.

The catalog published by Hill et al. (1982) contains about 800 stars with reliable photometric distances. For about 450 stars of this list we have found accurate parallaxes from HIPPARCOS. Figure 2 compares photometric and HIPPARCOS distances. As seen from the figure, up to $300 \mathrm{pc}$ the photometric distances tend to be smaller than the distances derived from parallaxes. This is revealed by the median line, calculated by binning photometric distances within $50 \mathrm{pc}$ intervals. The median deviates from the line of the equal distance starting from about $100 \mathrm{pc}$ and this deviation increases toward the larger distances. This trend is in the sense expected from the Malmquist bias of the 2nd kind (Teerikorpi 1997), due to the magnitude limit which at a fixed true distance cuts away stars from the faint side of the luminosity function. 


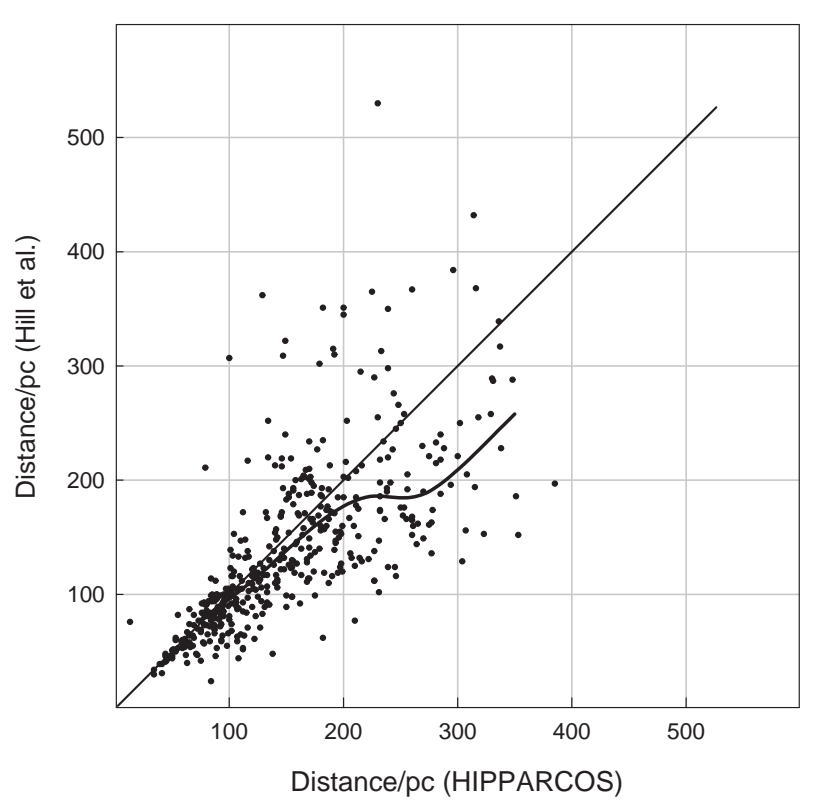

Fig. 2. Comparison of the photometric (Hill et al. 1982) and HIPPARCOS distances for the stars in the NGP area. The curve gives the median within $50 \mathrm{pc}$ intervals.

\subsection{Sampling}

Our polarimetry catalog contains 336 stars of spectral type from late-B to early-K located between latitudes $70^{\circ}-90^{\circ}$. In order to evaluate the completeness of our sampling we have used the photometric catalog published by Hill et al. (1982). It includes about 800 stars of A-F spectral types at $b>75^{\circ}$ with "reliable" photometric estimates of distance. Our catalog has 173 stars from Hill's list. The histogram in Fig. 3 represents the distribution of stars from Hill's catalog within three distance ranges, both for all stars and for those for which polarization has been measured. It is seen that the stars closer than $100 \mathrm{pc}$ and farther than $300 \mathrm{pc}$ are better represented in the polarimetry list than those at distances $100<d<300 \mathrm{pc}$. This selection effect is easy to understand: the new NOT observations were primarily concerned with faint and distant stars $\left(m_{V}>10.5\right)$, while the older measurements mostly dealt with nearby bright stars. This leaves the important range 100-300 pc much undersampled. Since there are some interesting structures seen in polarization at these distances (see below), we plan to complement our data in future with stars between 100 and $300 \mathrm{pc}$.

\subsection{Polarization data}

Polarization data taken from Markkanen (1979), Haikala (1979), Mathewson \& Ford (1970) and Korhonen \& Reiz (1986) were obtained in Johnson's $B$-band. Observations published by Appenzeller (1968) were obtained without filters, in white light. The data from Tuorla, Crimea and NOT were measured in Johnson's $U B V R I$ phometric system. In many cases, the accuracy of polarization measurements in $U$ and $I$ bands, due to the relatively poor photon

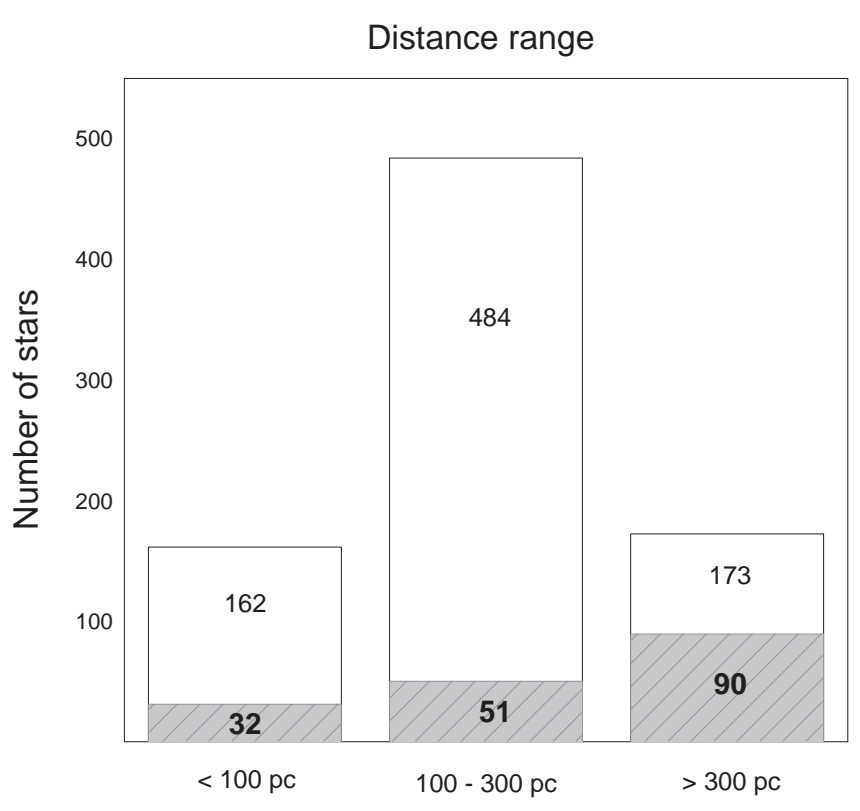

Fig. 3. Sampling of stars with the distance from the Hill et al. 1982 catalog. The number of stars with observed polarization is shown as the shaded portion of the corresponding bar.

statistics, was not good enough to meet the criterium we have adopted. The value of polarization we give in the catalog was measured in $B, V$ or $R$ depending on which passband the $P / \sigma_{\mathrm{P}}$ ratio is the highest. For about 30 stars the accuracy in all passbands is good enough to inspect the wavelength dependence of polarization. Examples of typical polarization spectra are shown in Fig. 4. Unfortunately, as the polarization is very low indeed, we cannot measure it with a high level of confidence through different wavelengths. Nevertheless, we have not found significant variations of polarization within the $U B V R I$ bands. In most cases the polarization spectrum has a moderate maximum in the wavelength range $400-700 \mathrm{~nm}$. The differences in $B$, $V$, and $R$ polarizations are small and seem to be within the measuring accuracy. The angle of polarization does not vary within wavelength range. This justifies our use of the band where the accuracy is the highest. We hope that in our new programme on the interstellar polarization around the south galactic pole, where the polarization seems to be higher (Berdyugin \& Teerikorpi 2001), we can better address the question of wavelength dependence at high galactic latitudes.

\subsection{Towards a more complete polarization map}

Figure 5 shows the map of interstellar polarization around the NGP. In comparison with the map given in Paper III this one contains 22 more stars from the list published by Mathewson \& Ford (1970). As seen from the map, the distribution of polarization vectors over the studied area is strongly non-random and reveals several distinct zones with differing orientation of Galactic magnetic field lines.

The most distinctive feature of the map is the "Markkanen's cloud" - the area of well aligned 

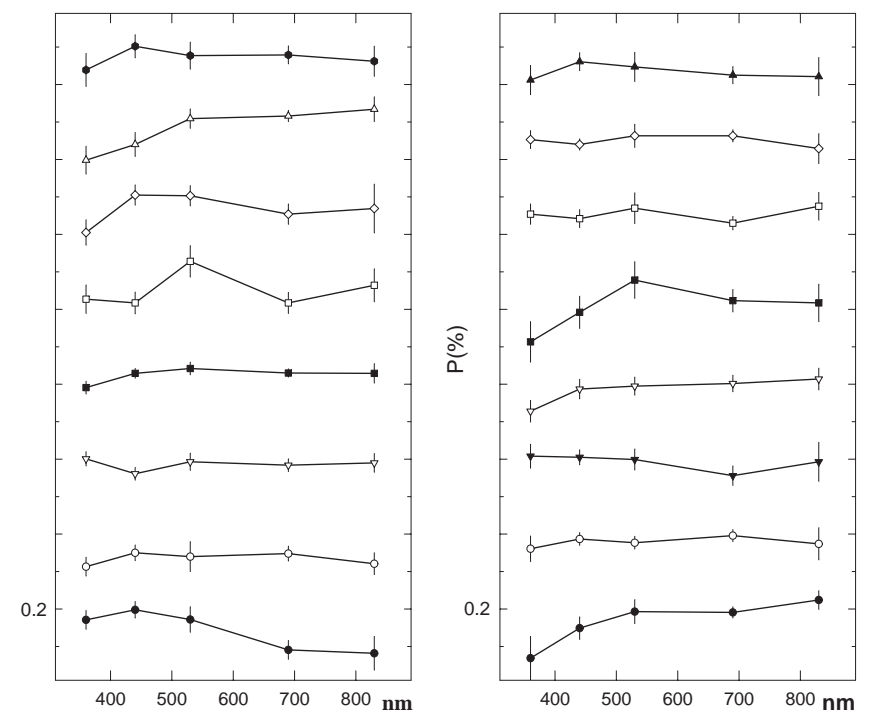

Fig. 4. Polarization of the selected stars plotted against the effective wavelengths of the $U B V R I$ passbands $(360,440,530$, 690 and $830 \mathrm{~nm})$. Vertical bars are $2 \sigma$ errors.

polarization vectors located approximately between the longitudes $240^{\circ}<l<30^{\circ}$ and the latitudes $70^{\circ}<b<$ $85^{\circ}, 70^{\circ}<b<75^{\circ}$. The magnitude of polarization in this part of the sky approches $0.45 \%$ which implies a lower limit for the interstellar extinction $A_{v} \geq 00^{\mathrm{m}} 13$. Markkanen's cloud starts at the distance of about $100 \mathrm{pc}$, extending well beyond of 300 pc. In his original work, Markkanen (1979) mapped the cloud up to $200 \mathrm{pc}$.

Another feature is the more distant $(d>300 \mathrm{pc})$ cloud located at $180^{\circ}<l<210^{\circ}$ and $75^{\circ}<b<83^{\circ}$. Polarization vectors in this cloud are directed roughly perpendicular to those in Markkanen's cloud. This implies that the projections of the magnetic fields on the tangential plane of the sky are perpendicular. The magnetic fields in these two regions could be perpendicular to each other, if the field is roughly parallel to the galactic plane. Both clouds are revealed on the IRAS 100 micron and ROSAT soft X-ray $(0.25 \mathrm{keV})$ maps. This finding and the NGP polarization map are discussed in detail in Paper III.

The catalogue of the polarization data is presented in Table $1^{1}$. The description of the catalog entries follow.

Column 1: Star identification. Whenever possible we give the BD (Bonner Durchmusterung) number. Some stars have Kn number,- e.g. Knude (1996) or ss2 number, - e.g. Sletterback \& Stock (1959).

Column 2: $P$, the polarization percentage. The following number in brackets gives the source of data: 1,- from the Crimea-Tuorla-NOT programme; 2,- Appenzeller (1968); 3,- Markkanen (1979); 4,-- Haikala (1979); 5,Korhonen \& Reiz (1986); 6,-- Mathewson \& Ford (1970).

Column 3: $\sigma_{\mathrm{P}}$, the error of polarization measurement.

1 Table 1 is only available withing the on-line version of A\&A and in electronic form at the CDS via anonymous ftp to cdsarc.u-strasbg.fr $(130.79 .128 .5)$ or via http://cdsweb.u-strasbg.fr/cgi-bin/qcat?J/A+A/372/276

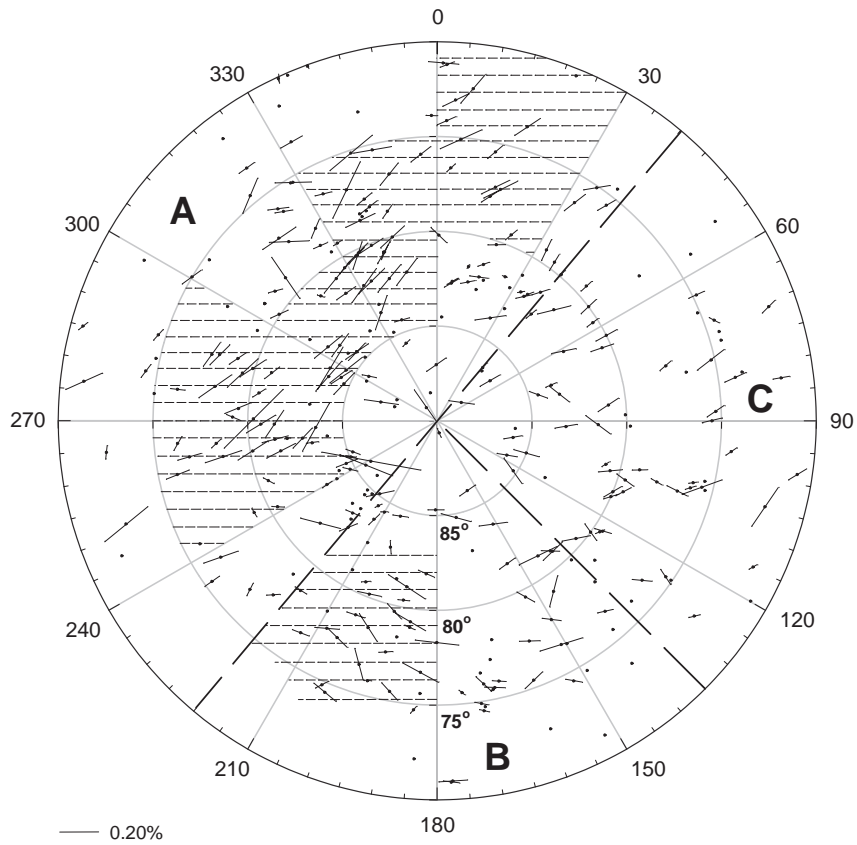

Fig. 5. Polarizations of the stars around the NGP. The length of the bar gives the amount of polarization, its direction gives the direction of the polarization plane. The dots are stars with very small/zero polarization. The sectors $\mathrm{A}, \mathrm{B}$, and $\mathrm{C}$ are separated by the dashed lines having the longitudes $40^{\circ}, 135^{\circ}$, and $220^{\circ}$. The shaded areas show the approximate location of Markkanen's cloud and of the distant cloud in sector B.

Column $4: \theta$, the position vector of polarization measured in the equatorial coordinate frame.

Column 5: $\sigma_{\theta}$, the error of position angle measurement.

Column 6: $d(\mathrm{pc})$, star distance, in parsecs, 1,- from Hill et al. (1982); 2,--from HIPPARCOS (Perryman et al. 1997); 3,- Knude 1996. Wherever possible we are giving the distance available from HIPPARCOS referring to other sources only if there is no accurate estimation of the parallax.

Columns 7 and 8: Galactic longitude and latitude, $\left(l^{\mathrm{II}}, b^{\mathrm{II}}\right)$.

Column 9: Sp, the spectral type, if available.

Column 10: the HD and SAO numbers, if available.

Column 11: Notes, here we give the additional information on the star: possible variability and, if available, alternative distance or polarization values.

Acknowledgements. The present work has been supported by the Academy of Finland (project "Cosmology in the local galaxy universe"). A. B. acknowledges the Väisälä Foundation for financial support, and also thanks warmly Prof. I. Tuominen for his kind support which the author received working at the University of Oulu.

This work is partly based on observations made with the Nordic Optical Telescope, operated on the island of La Palma jointly by Denmark, Finland, Iceland, Norway, and Sweden, in the Spanish Observatorio del Roque de los Muchacos of the Instituto de Astrophysica de Canarias. 


\section{References}

Appenzeller, I. 1968, ApJ, 151, 907

Berdyugin, A., Snåre, M.-O., \& Teerikorpi, P. 1995, A\&A, 294, 568, Paper I

Berdyugin, A., \& Teerikorpi, P. 1997, A\&A, 318, 37, Paper II

Berdyugin, A., Teerikorpi, P., \& Haikala, L. 2000, A\&A, 358, 717, Paper III

Berdyugin, A., \& Teerikorpi, P. 2001, A\&A, 368, 635

Haikala, L. 1979, unpublished pro graduate-study, University of Helsinki

Heiles, C. 1996, ApJ, 462, 316
Hill, G., Barnes, J. V., \& Hilditch, R. W. 1982, Publ. Dominion Astrophys. Obs., XVI, No. 10

Perryman, M. A. C., Lindegren, L., Kovalevsky, J., et al. 1997, A\&A, 323, L49

Knude, J. 1996, A\&A, 306, 108

Korhonen, T., \& Reiz, A. 1986, A\&AS, 64, 487

Markkanen, T. 1979, A\&A, 74, 201

Mathewson, D. S., \& Ford, V. L. 1970, Mem. Roy. Astr. Soc., 74,139

Slettebak, A., \& Stock, J. 1959, Astron. Abh. Hamburg. Sternw., 5, 105

Teerikorpi, P. 1997, ARA\&A, 35, 101 